assessment based on such data is a major source of bias in the risk estimation.

Methods A validation study was conducted based on a case-control study including 94 acoustic neuroma cases and 191 matched controls from the German InterphoneStudy to investigate the level of agreement between self-reported occupational noise exposure and a job-exposure-matrix (JEM) on noise exposure derived from a lifetime occupation calendar. The JEM was generated based on measurement data collected in German and Swiss literature for various occupations. Level of agreement was investigated by using sensitivity, specificity, $\kappa$ coefficient and the Youden Index.

Results As a result of a receiver operating characteristics analysis we dichotomise noise exposure at 80 decibel(Acoustic) $(\mathrm{dB}(\mathrm{A}))$, displaying a moderate agreement between self-reported exposure and the JEM-based exposure ( $\kappa$ of 0.53 ) that was slightly higher for cases than controls ( $\kappa$ of 0.62 and 0.48 ). The agreement was best for the loudest job reported and slightly lower for the longest held job or the last held job of the lifetime job history.

Conclusions The cut point of $80 \mathrm{~dB}(\mathrm{~A})$ corresponds with EU-regulations for workers. The levels of agreement between self-reported high occupational noise exposure compared with JEM-data, together with no substantial differences between cases and controls, suggest that selfreported data on occupational noise exposure is a valid exposure metric. Noise exposure appears to be appropriate if only exposure information on last or longest held job is available.

\title{
VALIDITY OF SELF-REPORTED OCCUPATIONAL NOISE
} EXPOSURE

Klaus Schlaefer, Brigitte Schlehofer, ${ }^{1}$ Joachim Schüz ${ }^{2}$ 'DFKZ, Heidelberg, Germany; 2IARC, Lyon, France

10.1136/oemed-2011-100382.137

Objectives In epidemiological studies the validity of self-reported occupational data is an important issue as the exposure 